\title{
Entropy Production and the Origin of Life
}

\author{
Karo Michaelian \\ Instituto de Física, Universidad Nacional Autónoma de México, Cto. de la Investigación Científica, \\ Cuidad Universitaria, Mexico City, Mexico \\ E-mail: karo@fisica.unam.mx \\ Received January 28, 2011; revised April 22, 2011; accepted April 25, 2011
}

\begin{abstract}
All irreversible processes arise and persist to produce entropy. Entropy production is not incidental to such processes, but rather the very reason for their origin and persistence. Here we take such a thermodynamic perspective on the origin of life, recognizing that entropy production is not only the vital force of life, but the fundamental link between life in the biosphere today and its origin in the Archean. Today the greatest entropy production in the biosphere is due to visible photon absorption and dissipation into heat by organic material in liquid water and the subsequent degradation of the established heat gradient through the water cycle. Following this link back in time to the Archean environment leads to a suggestion for a mechanism for the origin of life based on UV photon absorption and dissipation by RNA and DNA.
\end{abstract}

Keywords: Origin of Life, Entropy Production, RNA, DNA, Non-Equilibrium Thermodynamics, UVTAR

\section{Introduction}

In 1871, Charles Darwin in a private letter to his friend, the English botanist and explorer Joseph D. Hooker, suggested that life may have had a chemical origin [1]. However, for lack of an in depth analysis, or perhaps to avoid perturbing the conservative public of his time, Darwin wrote in his 1859 book "On the Origin of Species" that, "God first blew life into one or a few forms, and then evolution took over". One hundred years later, inspired by Oparin's 1924 suggestion of a material origin of life [2], Miller and Urey [3] showed that subjecting what was then the best hypothesis for the gasses of the prebiotic atmosphere (methane, ammonia, water, and carbon dioxide) to electric discharge was sufficient to produce at least 11 of the 20 then known amino acids making up the proteins of life (actually there are 22 known amino acids of life). Since then, many other similar experiments have demonstrated abiogenic routs to, not only the amino acids, but also to the nucleic acid bases, the ribose-like sugars, and the polyphosphates; the basic constituents of RNA and DNA, the probable first molecules of life $[4,5]$.

Although both single and double strand RNA and DNA can now be readily abiotically synthesized and manipulated in vitro, more than 50 years of experimentation since the first Miller experiments has been insufficient to produce a replicating life de novo in the labora- tory. The difficulty has been to demonstrate self-replication of RNA or DNA without the aid of the enzymes and the free energy containing molecules such as ATP. Today, in even the simplest archaea and bacteria, replication is carried out with the aid of a large number of enzymes, encoded for in the DNA and produced in the ribosome organelles of the complex cell. How then could RNA or DNA replicate, at the very beginnings of life, without the availability of these enzymes and the free energy containing molecules? This problem has been studied extensively with limited progress to date [4,5]. The most promising avenues led to the premise that RNA was the first molecule of life, which led to a paradigm now known as the "RNA World". Indications that RNA might have played a part in its own replication are various; 1) the observed ability of RNA to self-splice [6], 2) to act as a rybozyme; i.e. as a polymerase catalyst in template assisted polymerization of itself $[7,8]$, and 3 ) its ability to act as a catalyst for the formation of peptide bonds between amino acids [9].

Many theories have been proposed, and experiments performed, in the search for the origin of life (for an overview of the recent history see Kumar [10]). Most experiments have considered near-equilibrium conditions in the presence of mineral or clay catalysts, and look for the appearance of an auto-catalytic chemical cycle. However, the expectation of observing the origin of an irreversible process such as life, without due considera- 
tion, or even recognition, of its thermodynamic function of entropy production, is erroneous. All irreversible processes arise and persist to produce entropy. This is true of the burning flame of a candle, hurricanes, the water cycle, and, of course, life itself. Irreversible processes will arise, couple, and persist whenever the global entropy production of the Universe increases, and as long as all natural laws and constants are respected [11]. Boltzmann [12] (1886) understood the implications of this when, only 27 years after the publication of "On the Origin of Species", he wrote "The general struggle for existence of animate beings is therefore not a struggle for raw materials - nor for energy which exists in plenty in any body in the form of heat - but a struggle for entropy, which becomes available through the transition of energy from the hot sun to the cold earth". Entropy production is not incidental to the process of life; the process of life obtains its vitality and reason for being through entropy production.

If entropy production is the driving force of life, then this, of course, must have been the case since its very beginnings. Entropy production is thus a link that permeates the entire evolutive history of life on Earth. It is then of interest to follow this link back in time, starting from the mechanism of entropy production employed by life today and searching for an analogy which could have been operating at the very beginnings of life, involving the probable first molecules of life, RNA and DNA, and the prevalent ambient conditions. Such logic leads to a new paradigm for the origin of life, consistent with what we have learned so far, but incorporating these new thermodynamic elements which lend direction to life's origin.

\section{The Thermodynamic Function of Life}

By far the most abundant biomass at the surface of the Earth consists of plants and cyanobacteria [13]. Phototrophic plants and cyanobacteria over land and water have traditionally been considered as the first link of a food chain, or, as the base of an ecological pyramid with the highest predators residing at its pinnacle. However, from a thermodynamic viewpoint, plants and cyanobacteria do much more than supply the rest of life with free-energy rich organic materials obtained through photosynthesis. In fact, all photosynthetic production uses less than $0.1 \%$ of the free energy available in sunlight absorbed by the leaves of the plant [14] or absorbed by cyanobacteria. By far the greatest amount of free energy in sunlight incident on the plant is consumed in transpiration. Water is drawn up by the roots and evaporated from the leaves of plants and thereby delivered into the global water cycle. On the ocean surface and over moist land surfaces, cyanobacteria absorb sunlight and dissipate this light into infrared wavelengths that can be readily absorbed by water, thereby increasing the evaporation from the oceans and land and thus also contributing to the global water cycle [15].

Animals, by degrading the free energy available in plant and cyanobacterial matter, also augment the entropy production of the Earth in its interaction with its solar environment. However, this direct entropy production is insignificant compared to the increase in entropy production afforded indirectly to the plants through their interaction with animals. Animals stimulate plant growth and dissemination by spreading nutrients and seeds, and by providing a vehicle for cross fertilization. Their digging and stirring brings oxygen and nitrogen into the soil and water, impacting significantly primary productivity. The most important thermodynamic function of the animals is therefore to ensure that the plants and cyanobacteria are well cared for and able to spread into new areas, thereby attaining maximal entropy production through photon dissipation coupled to evapotranspiration [15].

\section{Entropy Production Due to Life Today}

The plants and cyanobacteria of today absorb light over a wide range of the most intense part of the Sun's spectrum. Although the chlorophyll (a) and chlorophyll (b) molecules have narrow absorption spectra, peaking around $430 \mathrm{~nm}$ (in the blue) and $662 \mathrm{~nm}$ (in the red), there exist many accessory pigments, or antenna type molecules such as the carotenoids, flavonoids, and betalains, that allow the plant to absorb over a much wider range of the solar spectrum. These accessory pigments appear to have little physiological role in photosynthesis or in plant metabolism and in fact release most of the absorbed light energy as heat rather than in chemical transformations. Even the absorption of light by chlorophyll itself is subjected to many non-photochemical quenching routs having nothing to do with photosynthesis [16]. This leads to the interesting result that the photo-absorption spectrum of both plants and cyanobacteria is significantly more extent than the photo-activation spectrum of photosynthesis.

Recently, a large variety of related molecules known as microsporine-like amino acids (MAAs), which absorb over the UVA and UVB region of the Sun's spectrum, have also been discovered in plants and phytoplankton [17]. A single plant may contain many MAAs, leading to an approximately flat absorption spectrum in the near ultraviolet. The existence of these pigments and antenna type molecules has hitherto been assigned as rudiments of the evolutionary development of the photosynthetic apparatus [18], principally as agents that protect (or, 
protected) the photosynthetic apparatus from damage caused by an excess of photons, or photons of very high energy. However, since photosynthesis contributes little to the total entropy production of plants or cyanobacteria, while transpiration contributes overwhelmingly, a more likely thermodynamically consistent explanation is that such pigments and antenna molecules are still very relevant, acting primarily to aid in photon dissipation and transpiration, the primary entropy producing process of a plant or cyanobacteria.

An interesting experimental result corroborating this idea - that the primary thermodynamic function of life today is entropy production through photon dissipation and transpiration - has been obtained by Wang et al. [19]. They find vanishing derivatives of transpiration rates with respect to leaf temperature and $\mathrm{CO}_{2}$ flux, suggesting a maximum transpiration rate in these variables for plants. In fact, they found that the particular partition of latent and sensible heat fluxes to be such that it leads to a leaf temperature and leaf water potential giving maximal transpiration rates, and thus maximal production of entropy. Although such an optimality principle has frequently been suggested to be operating for the process of photosynthesis, to the author's knowledge, no empirical evidence has so far confirmed this. Plants, therefore, appear to have evolved for optimizing transpiration rather than photosynthesis.

\section{Entropy Production Due to Life in the Archean}

Can entropy production through photon dissipation and the subsequent transpiration of water by life today be traced back in time to the very beginnings of life? Since life is an open irreversible process dependent on external thermodynamic forces (in particular, the solar photon flux), to answer this, it is first necessary to determine the environmental conditions existing at the surface of the Archean Earth some 3.8 billion years ago. A discussion of the most probable, given present knowledge, ambient conditions of the prebiotic Earth relevant to the beginnings of life have been given elsewhere [20]. Here we summarize only the most important aspects relevant to the proposed hypothesis.

The surface of the Archean Earth was initially very hot, due to the heat of accretion, high internal radioactivity, and asteroid bombardment. The surface temperature fell to roughly $(70 \pm 15)^{\circ} \mathrm{C}$ during the $3.5-3.2 \mathrm{Ga}$ era [21], as determined from geochemical evidence in the form of ${ }^{18} \mathrm{O} /{ }^{16} \mathrm{O}$ ratios found in cherts of the Barberton greenstone belt of South Africa. The atmosphere was probably composed of nitrogen, carbon dioxide and reducing gases containing a lot of hydrogen. The photon absorp- tion spectrum of such an atmosphere, taking into account the aldehydes formed by UV photochemical reactions on these gases [22], would have lead to an atmospheric window of transparency in the ultraviolet of between approximately $240 \mathrm{~nm}$ and $290 \mathrm{~nm}[20,22]$. In fact, detailed simulations of the Archean atmosphere, considering the light absorption and scattering properties of these gases, as well as the fact that the young Sun was much more intense in the ultraviolet during the Archean, show that the amount of ultraviolet light at $254 \mathrm{~nm}$ reaching the Earth's surface during the Archean could have been up to $10^{31}$ times that of what it is today [23].

The high surface temperature of the Archean Earth would also imply a greater amount of water vapor in the atmosphere than today. More prevalent volcanic eruptions would also mean a larger amount of sulfur dioxide in the atmosphere. UV photochemical reactions on these gases would have produced a thin layer of sulfuric acid clouds that would have been highly reflective in the visible, as on Venus today. It is then probable that an enthropically important part of the Sun's spectrum reaching the surface of the Earth would have been that in the ultraviolet between roughly $240 \mathrm{~nm}$ and $290 \mathrm{~nm}$.

Now, it is an interesting fact that the nucleic acid bases, adenine, thymine, cytosine, guanine, and uracil absorb very strongly just at these wavelengths (peak absorption of DNA at $260 \mathrm{~nm}$ ) and in water dissipate the photoninduced excitation energy directly and rapidly $\left(10^{-12} \mathrm{~s}\right)$ to heat (vibrational and rotational molecular motion) of the surrounding water [24-27] (see Figure 1). The nucleic acid bases are thus excellent entropy producing molecules in the ultraviolet region of the Sun's spectrum. During the Archean, these molecules would have played a thermodynamic role analogous to the chlorophyll molecule and other pigments of today in dissipating the high energy photons reaching the Earth's surface and catalyzing the water cycle.

Under the very high flux of UV light prevalent during the Archean, the absorption and rapid non-radiative decay characteristics in the ultraviolet of the nucleic acid bases would have given these a selective advantage over other organic molecules more prone to photolysing or photochemical reactions [22]. The nucleic acid bases would then fair better in the competition for the more basic constituent molecules needed for their synthesis. Other molecules which could attach themselves to the nucleic acid bases would also obtain, by association, significant UV protection, and thus selective advantage [28]. Such molecules might have included adenosine triphosphate (ATP) and the five nucleotides. However, over and above the selective advantage of UV protection, the important ability of these molecules to absorb and dissipate into heat a high energy photon would promote 


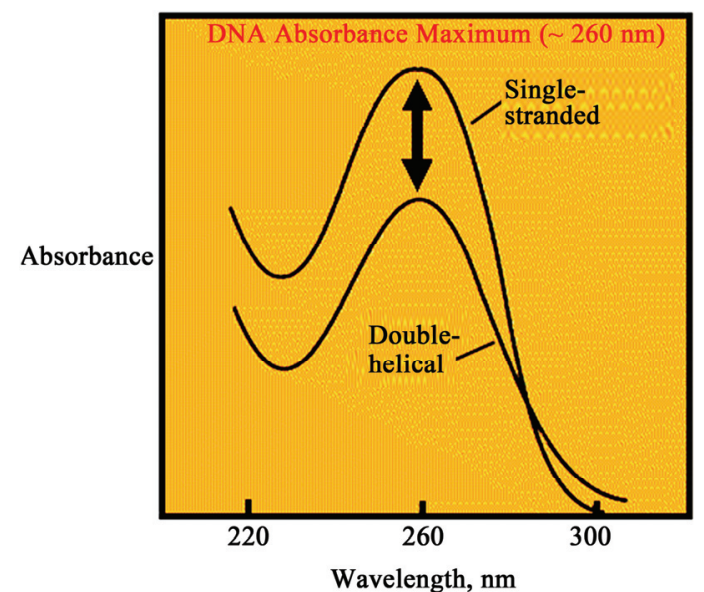

(a)
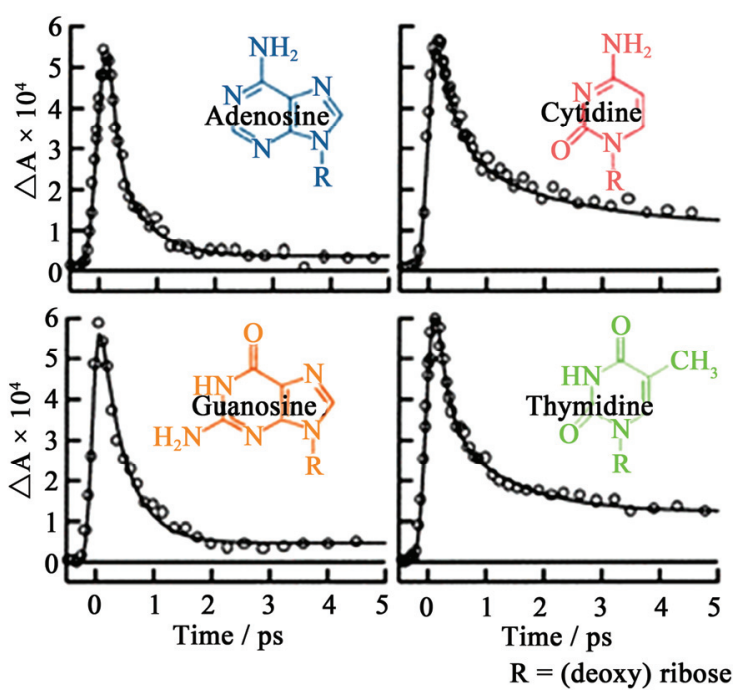

(b)

Figure 1. (a) Absorption maximum in the ultra violet at $260 \mathrm{~nm}$ of single strand and double helix DNA. The difference, marked by the double arrow, is known as hypochroism and is due to the random orientation of the bases in single strand DNA (or RNA); (b) Rapid non-radiative decay of the excited bases of DNA when in water (within a pico-second). Reproduced with permission from Pecourt et al. [27].

their existence and proliferation as a thermodynamic imperative. A non-equilibrium chemical rout to the production, maintenance and multiplication of these molecules would have been sought out by Nature simply due to their entropy producing characteristics in the intense UV environment of Archean Earth [20].

\section{Multiplication of RNA and DNA without the Aid of Enzymes}

Molecules containing the UV absorbing and dissipating nucleic acid bases would thus gradually have accumu- lated on the surfaces of the shallow seas; their survival being favored by their rapid and non-radiative excited state decay dynamics, and their multiplication being favored by the increase in entropy production afforded to the Earth within its solar environment. However, the most difficult question remains: Just how could their multiplication have occurred without the aid of enzymes or free energy containing molecules? Here I suggest that the ambient conditions of Archean Earth could have played an important part in the replication of primordial RNA or DNA.

Given a large accumulation of nucleic acid bases and other derivatives of the bases, protected from UV lysing and photochemical reactions, at the sea surface, it is reasonable to expect at least some polymerization of the nucleotides. Polymerization would, in fact, be thermodynamically driven since UV excited single strand RNA and DNA is more apt to decay through rapid intersystem crossing channels, and therefore be less prone to photolysing or photoreactions, than are the isolated bases which can loose efficiency by decaying to a much longer lived ${ }^{1} n \pi^{*}$ state [29].

If the surface of the Earth was initially very hot, fal- ling to a temperature of $(70 \pm 15)^{\circ} \mathrm{C}$ during the $3.5-3.2 \mathrm{Ga}$ era [21], then it is probable that just before the very beginnings of life at approximately $3.8 \mathrm{Ga}$, the surface temperature of the seas would have been above the denaturing temperature of RNA and DNA (generally around $85^{\circ} \mathrm{C}$, but dependent on the amount of guaninecytosine pairs, which tend to increase the denaturing temperature). Continued cooling of the seas would have led to a situation at which the surface temperature of the sea at night would have been below the denaturing temperature of at least some segments of RNA and DNA, allowing these segments to act as templates for the construction of a complimentary segment overnight. As the sun rose, the surface of the seas would again heat up through ultraviolet, visible, and infrared light absorption, such that by late afternoon the temperature of the surface water would be sufficient to denature double strand RNA or DNA. In this way, new templates would be formed for continued replication. An important part of the heating of the surface water in the local neighborhood of the double strand RNA/DNA would have come from the direct absorption and rapid dissipation of UV photons by the bases within the 240 to $290 \mathrm{~nm}$ region [20].

The day-night temperature cycling of the sea surface around the denaturing temperature of RNA/DNA, along with the direct absorption and dissipation of a UV photon, would have provided a mechanism for replication similar to that which is in general use today in the laboratory, known as "polymerase chain reaction" [30]. The mechanism postulated here for early replication may be called 
"UV and Temperature Assisted Replication (UVTAR)" and is depicted in Figure 2.

As the seas continued to cool, those RNA/DNA segments with lower denaturing temperature, or with sections coding for a simple denaturing enzyme, could continue replicating while the rest of the double strands would become locked, unable to denature, and thus be effectively removed from competition for the nucleotides. Coding for a simple denaturing enzyme may thus have been the first utility of information storage for RNA or DNA. As the seas cooled further, continued entropy production would require the natural selection of RNA or DNA segments coding for still more complex enzymes that could perform the denaturing at still cooler temperatures, or for antenna type molecules that could dissipate more of the solar spectrum and deliver the resultant heat locally. RNA/DNA segments that coded for enzymes that could attract and then polymerize nucleotides on existing strands (for example, polymerase-type) would become more favored still as the seas cooled further.

Thus may have begun the first steps of evolution through natural selection. The tendency of Nature to discover new routs to greater entropy production, often building on existing routs, under the imposed solar photon flux over the Earth, was, and still is, the driving force behind evolution. Entropy production in the early days of life was afforded by the remarkable absorption and dissipation characteristics of single strand RNA/DNA existing in abundance at the surface of the seas exposed to very intense UV radiation. As the hydrogen containing prebiotic molecules gradually became depleted from the Archean atmosphere, the skies would have begun to clear of the aldehydes and sulfuric acid clouds, allowing more visible radiation to penetrate to the surface. The normal evolution of solar type stars implies a Sun brightening in the visible and dimming in the ultraviolet. Further increases in entropy production would thus have come from dissipating more light towards the visible region of the spectrum. This would have meant the gradual selection of RNA or DNA coding for pigments that absorbed and dissipated in the UVA and UVB spectrum, such as the microsporine pigments based on the most readily abiogenically synthesized amino acid glycine, and eventually pigments in the visible, such as the chlorophyll molecule, based on the porphyrins.

In contrast to the RNA-first theory on the origin of life, in the present theory, information content and fidelity of replication were not prerequisites to the beginning of life since replication was afforded by the UVTAR mechanism without the need for enzymes, at least while environmental conditions were favorable. However, as the ambient conditions at the Earth's surface moved ever further from those relevant for UVTAR, information and fidelity of replication became ever more important to the maintenance and multiplication of the photon dissipating molecules, and thereby to global entropy production of Earth.

\author{
UV and Temperature Assisted \\ Reproduction (UVTAR) of RNA/DNA
}
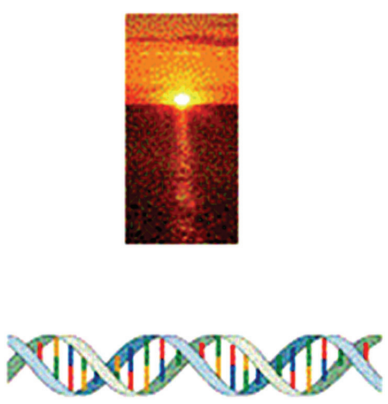

(a)
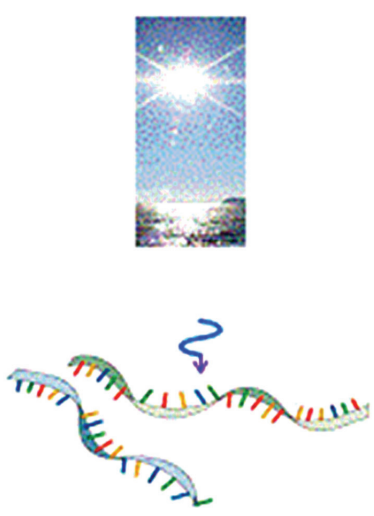

(b)
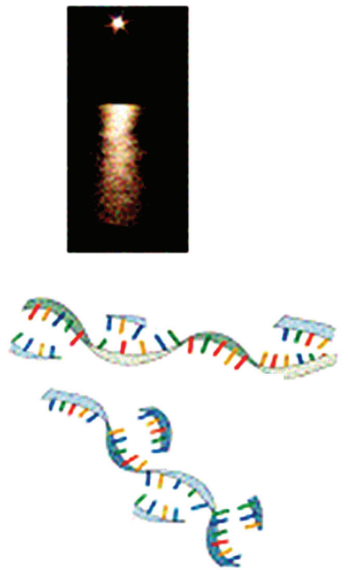

(c)

Figure 2. (a) Double strand RNA/DNA floats on the Archean sea surface in the early morning hours. (b) By late afternoon the sea surface temperature heats up beyond the denaturing temperature of RNA/DNA and the strands separate, in part due to the direct absorption of UV photons by RNA/DNA; (c) Over night, the sea surface temperature cools to below the denaturing temperature and single strand RNA/DNA act as template for the formation of a complimentary strand. The entire replication process is driven by entropy production resulting from the absorption and dissipation of UV photons. 


\section{Conclusions}

Entropy production is the link that permeates all of life from its humble beginnings in the Archean to the vast and complex ecosystems of today. Understanding this thermodynamic function of life has allowed us to trace this link back in time and suggest an analogous entropy producing mechanism involving the most probable first molecules of life, RNA and DNA. Absorption and dissipation of UV light around $260 \mathrm{~nm}$ was probably the primordial thermodynamic function of the first RNA or DNA single strand molecules floating on the ocean surface. The remarkable UV absorption and dissipation characteristics of the nucleic acid bases afforded the nucleotide polymers protection from photo-lysing and photochemical reactions, allowing them to accumulate to significant concentrations on the surfaces of the Archean seas. Their entropy producing function for the Earth in its solar environment promoted their maintenance and multiplication. As the temperature of the seas fell to below the denaturing temperature of certain RNA or DNA segments, these could begin to act as templates for the formation of complimentary strands during the cooler periods overnight. As the seas cooled further, those segments which happened to code for simple denaturing enzymes or antenna type molecules would find greater advantage in an ever colder sea. The cooling of the ocean surface was thus probably the force that stimulated the information content and corresponding reproductive fidelity of RNA and DNA.

As the light conditions of the Earth's surface changed, as a result of combined biotic, atmospheric, and solar evolution, entropy production was driven towards the dissipation of longer wavelengths of greater intensity closer to the visible region of the Sun's spectrum, which eventually led to the coding for chlorophyll and other contemporary pigments. The tendency towards evolution of new pigments covering an ever greater region of the Sun's spectrum remains to this day; old conifer forests of climax ecosystems are almost black and absorb and dissipate more incident light than any other biotic or abiotic system on the surface of the Earth. The primary function of animals is to facilitate the maintenance and spread of the photon dissipating pigments throughout the surface of the Earth.

The origin of life was contingent on the increase in the entropy production it afforded Earth under the existent environmental conditions. Tracing the link of entropy production through photon dissipation and transpiration back in time to the Archean, where UV dissipation took precedent over visible dissipation, leads to, a thermodynamic reason for the origin of life on Earth.

\section{Acknowledgements}

The financial assistance of DGAPA-UNAM, grant numbers IN118206 and IN112809, is greatly appreciated.

\section{References}

[1] J. Pereto, J. L. Bada and A. Lazcano, "Charles Darwin and the Origin of Life," Origins of Life and Evolution of Biospheres, Vol. 39, No. 5, 2009, pp. 395-406. doi:10.1007/s11084-009-9172-7

[2] A. I. Oparin, "The Origin of Life," Moscow Worker Publisher, Moscow, 1924; A. I. Oparin, Translate, "The Origin and Development of Life," NASA TTF-488, Washington DC, 1968.

[3] S. L. Miller and H. C. Urey, "Organic Compound Synthesis on the Primitive Earth," Science, Vol. 130, No. 3370,1959 , pp. 245-251. doi:10.1126/science.130.3370.245

[4] L. E. Orgel, "Prebiotic Chemistry and the Origin of the RNA World," Critical Reviews in Biochemistry and MoLecular Biology, Vol. 39, No. 2, 2004, pp. 99-123. doi:10.1080/10409230490460765

[5] H. Rauchfuss, "Chemical Evolution and the Origin of Life," Springer, New York, 2008.

[6] K. Kruger, P. J. Grabowski, A. J. Zaug, J. Sands, D. E. Gottschling and T. R. Cech, "Self-Splicing RNA: Autoexcision and Autocyclization of the Ribosomal Rna Intervening Sequence of Tetrahymena," Cell, Vol. 31, No. 1, 1982, pp. 147-157. doi:10.1016/0092-8674(82)90414-7

[7] W. K. Johnston, P. J. Unrau, M. S. Lawrence, M. E. Glasner and D. P. Bartel, "RNA-Catalyzed RNA Polymerizetion: Accurate and General RNA-Templated Primer Extension," Science, Vol. 292, No. 5520, 2001, pp. 13191325. doi:10.1126/science. 1060786

[8] H. S. Zaher and P. J. Unrau, "Selection of an Improved RNA Polymerase Ribozyme with Superior Extension and Fidelity," Ribonucleic Acid, Vol. 13, 2007, pp. 1017-1026.

[9] B. Zhang and T. R. Cech, "Peptide Bond Formation by in Vitro Selected Ribozymes," Nature, Vol. 390, 1997, pp. 96-100. doi:10.1038/36375

[10] A. L. Kumar, "Origin of Life," Astrophysics and Space Science, Vol. 317, No. 3-4, 2008, pp. 267-278. doi:10.1007/s10509-008-9876-6

[11] I. Prigogine, G. Nicolis and A. Babloyantz, "ThermodyNamics of Evolution (I)," Physics Today, Vol. 25, No. 12, 1972, pp. 23-28; "Thermodynamics of Evolution (II)," Physics Today, Vol. 25, No. 12, 1972, pp. 38-44. doi:10.1063/1.3071140

[12] L. Boltzmann, "The Second Law of Thermodynamics," In: B. Mcginness, Ed., Ludwig Boltzmann: Theoretical Physics and Philosophical Problems: Selected Writings, Reidel Publishing Co., Dordrecht, 1974.

[13] W. B. Whitman, D. C. Coleman and W. J. Wiebe, "ProKaryotes: The Unseen Majority," Proceedings of the $\mathrm{Na}$ tional Academy of Sciences, Vol. 95, No. 12, 1998, pp. 
6578-6583. doi:10.1073/pnas.95.12.6578

[14] D. M. Gates, "Biophysical Ecology," Springer-Verlag, New York, 1980.

[15] K. Michaelian, "Biological Catalysis of the Hydrological Cycle: Life's Thermodynamic Function, Hydrology and Earth System Sciences Discussions, Vol. 8, 2011, pp. 1093-1123, doi:10.5194/hessd-8-1093-2011

http://www.hydrol-earth-syst-sci-discuss.net/8/1093/2011/h essd-8-1093-2011.html; and "Thermodynamic Function of Life," General Physics, 2009, Arxiv: 0907.0040v2.

[16] P. Horton, A. V. Ruban and R. G. Walters, "Regulation of Light Harvesting in Green Plants," Plant Physiology, Vol. 106, 1994, pp. 415-420.

[17] K. Whitehead and J. I. Hedges, "Analysis of Mycosporine-Like Amino Acids in Plankton by Liquid Chromatography Electrospray Ionization Mass Spectrometry," Marine Chemistry, Vol. 80, No. 1, 2002, pp. 27-39. doi:10.1016/S0304-4203(02)00096-8

[18] V. B. Evstigneev, "On the Evolution of the Photosynthetic Pigments," Origins of Life, Vol. 6, No. 3, 1975, pp. 435-439. doi:10.1007/BF01130346

[19] J. Wang, R. L. Bras, M. Lerdau and G. D. Salvucci, "A Maximum Hypothesis of Transpiration," Journal of Geophysical Research, Vol. 112, 2007, Article ID G03010.

[20] K. Michaelian, "Thermodynamic Origin of Life," Earth System Sciences Discussions, Vol. 1, 2010, pp. 1-39; and "Thermodynamic Dissipation Theory for the Origin of Life," Earth System Dynamics, Vol. 2, No. 1, 2011, pp. 37-51.www.earth-syst-dynam.net/2/37/2011/ doi:10.5194/esd-2-37-2011

[21] D. R. Lowe and M. M. Tice, "Geologic Evidence for Archean Atmospheric and Climatic Evolution: Fluctuating Levels of $\mathrm{Co}_{2}, \mathrm{Ch}_{4}$, and $\mathrm{O}_{2}$ with an Overriding Tectonic Control," Geology, Vol. 32, No. 6, 2004, pp. 493-496. doi:10.1130/G20342.1

[22] C. Sagan, "Ultraviolet Selection Pressure on the Earliest Organisms," Journal of Theoretical Biology, Vol. 39, No.
1, 1973, pp. 195-200. doi:10.1016/0022-5193(73)90216-6

[23] I. Cnossen, J. Sanz-Forcada, F. Favata, O. Witasse, T. Zegers and N. F. Arnold, "The Habitat of Early Life: Solar X-Ray and Uv Radiation at Earth's Surface 4 - 3.5 Billion Years Ago," Journal of Geophysical Research, Vol. 112, 2007, Article ID E02008.

[24] D. Voet, W. B. Gratzer, R. A. Cox and P. Doty, “Absorption Spectra of Nucleotides, Polynucleotides and Nucleic Acids in the Far Ultraviolet," Biopolymers, Vol. 1, No. 3, 1963, pp. 193-208. doi:10.1002/bip.360010302

[25] P. R. Callis, "Electronic States and Luminescence of Nucleic Acid Systems," Annual Review of Physical Chemistry, Vol. 34, 1983, pp. 329-357. doi:10.1146/annurev.pc.34.100183.001553

[26] J. -M1. Pecourt, J. Peon and B. Kohler, "Ultrafast Internal Conversion of Electronically Excited RNA And DNA Nucleosides in Water," Journal of the American Chemical Society, Vol. 122, No. 38, 2000, pp. 9348-9349. doi:10.1021/ja0021520

[27] J.-Ml. Pecourt, J. Peon and B. Kohler, "DNA ExcitedState Dynamics: Ultrafast Internal Conversion And Vibrational Cooling in a Series of Nucleosides," Journal of the American Chemical Society, Vol. 123, No. 42, 2001, pp. 10370-10378. doi:10.1021/ja0161453

[28] A. Y. Mulkidjanian, D. A. Cherepanov and M. Y. Galperin, "Survival of the Fittest before the Beginning of Life: Selection of the First Oligonucleotide-Like Polymers by UV Light," Boston Medical Center Evolutionary Biology, Vol. 3, 2003, p. 12.

[29] C. T. Middleton, K. De La Harpe, C. Su, Y. K. Law, C. E. Crespo-Hernández and B. Kohler, "DNA Excited-State Dyanmics: From Single Bases to the Double Helix," Annual Review of Physical Chemistry, Vol. 60, 2009, pp. 217-239.

doi:10.1146/annurev.physchem.59.032607.093719

[30] K. Mullis, "The Unusual Origin of the Polymerase Chain Reaction," Scientific American, Vol. 264, No. 4, 1990, pp. 56-65. doi:10.1038/scientificamerican0490-56 